\title{
A Unifying Account of Angular Gyrus Contributions to Episodic and Semantic Cognition
}

\author{
Gina F. Humphreys ${ }^{1}$, Matthew A. Lambon Ralph ${ }^{1}$, and Jon S. Simons ${ }^{2}$
}

1. MRC Cognition and Brain Sciences Unit, University of Cambridge, UK

2. Department of Psychology, University of Cambridge, UK

Correspondence: Jon S. Simons, Department of Psychology, University of Cambridge, Downing Street, Cambridge CB2 3EF, UK. email: jss30@cam.ac.uk

Acknowledgements: Preparation of this article was supported by James S. McDonnell Foundation Scholar Award \#220020333, an MRC Programme grant (MR/R023883/1) and MRC intramural funding (MC_UU_00005/18) 


\section{$\underline{\text { Abstract }}$}

The angular gyrus (AG) region of lateral parietal cortex has been implicated in a wide variety of tasks and functions, generating numerous influential theories. However, these theories fail to explain why so many apparently distinct cognitive activities implicate common parietal structures. We propose a unifying model, based on a set of central principles, to account for coalescences of cognitive task activations across AG. To illustrate, we show how these principles account for findings from studies of episodic and semantic memory that have independently implicated the same AG regions but thus far been considered from largely domain-specific perspectives. We conclude that AG computations, as part of a wider lateral parietal system, enable the online dynamic buffering of multisensory spatio-temporally extended representations. (120 words) 


\section{Identifying Principles of Lateral Parietal Cortex Function}

Overview

In this review we evaluate the role of lateral parietal cortex, in particular angular gyrus (AG), in two related but distinctive memory systems: episodic and semantic memory. The AG has been implicated in a myriad of cognitive domains and numerous domain-specific theories of function have been proposed. Here we use evidence from episodic and semantic memory studies as a test case to examine the potential role of the AG (and, perhaps, by extension the wider LPC), in cognitive processes. We examine each literature in turn, as well as pointing out some key pitfalls that must be circumvented in order to more readily understand implications of the data. We propose a unified model in which AG, as part of a wider LPC system, operates as an online, dynamic, multi-sensory buffer that, through experience, becomes sensitive to the sequential spatio-temporal structure of an event or behaviour that unfolds over time.

The multi-task-multi-brain-region challenge

A key goal of cognitive neuroscience is to relate cognitive functions and dysfunctions to underlying neural processing. Functional neuroimaging, cognitive neuropsychology and other related neuroscience methods most commonly explore one specific domain (e.g., tasks pertaining to attention, episodic memory, language, etc.) and investigate which brain regions are implicated and how their function varies following changes to important task-related parameters. When considering this principal aim of cognitive-clinical neuroscience, a rapidly evolving challenge emerges: if we step beyond the large literature dedicated to each behavioural domain, then it becomes readily apparent that many neural regions are common to numerous, diverse behavioural activities, generating the conclusion that there is no simple one-to-one mapping between each type of activity and a single underlying brain region. Instead, each behavioural domain maps onto a cortical network and, in reverse, each cortical region seems to play a role in a variety of different cognitive behaviours. Broadly speaking, there are two contrastive approaches to this complex task-brain mapping conundrum. The first, which we might term "neuromarquetry" [1], assumes that functions/tasks are supported by a series of discrete, neighbouring subregions. Under this hypothesis, behaviour-brain mappings look complex because of the tight packing of functions into a brain region, yet are more one-to-one when considered at a finer spatial resolution. An alternative, the "primary systems hypothesis" [2-7], observes that cognitive tasks and activities are likely to be supported by variable combinations of more generalised neurocognitive computations and that these 'primary systems' will be called upon by multiple tasks. Under this view, the coalescence of task-related brain activity or dysfunctions in neuropsychological patients with respect to the same brain region, may reflect the shared neurocomputation that the tasks call upon. 
This multi-task-multi-brain-region challenge repeats across many brain regions and for a myriad of tasks - a complete description of which is beyond the scope of this brief review. Instead, we have selected a pertinent and prominent worked example: the lateral parietal cortex (LPC), centred on the AG, and its purported contributions to episodic and semantic memory. Like other tertiary association regions, the LPC has been implicated in a wide variety of tasks and functions [1, 8, 9]. We focus here upon AG contributions to episodic and semantic memory because (a) there are considerable bodies of functional neuroimaging and neuropsychology literature on both topics, (b) there are matured domainspecific theories and proposals about the AG in each domain, yet (c) these literatures have tended to remain isolated from each other despite being centred on two forms of long-term declarative memory and implicating the same LPC regions (although see recent reviews from [10,11]). Accordingly, these theories (and those for other cognitive domains) fail to unify the myriad of cognitive activities that implicate certain common LPC structures. We propose a unifying model, based a set of central principles, to account for the variety of cognitive tasks which activate parietal cortex. We illustrate how these central principles might account for the findings from cognitive neuroscience studies of episodic and semantic memory. For clarity we will define our anatomical labelling here. The LPC is a heterogeneous region with multiple graded subregions (see Box 1). Whereas some of the observations and proposals we identify may well apply more broadly across these LPC regions, in this review we focus principally upon the episodic and semantic memory data implicating AG, and differentiate between its dorsal subregion (PGa) that borders with the IPS, and the more ventral AG (PGp).

We focus here on three central principles. These notions link to a somewhat broader framework (the Parietal Unified Connectivity-biased Computation model [1, 7, 12] and previous seminal proposals $[8,13]$ which have taken a cross-domain perspective upon the AG (and wider LPC) and attempted to distil what generalised neurocomputations might be supported by this region and its long-range connectivity [9]. The first principle is that there are two orthogonal types of representations or statistical structures that can be extracted from our time-extended, multimodal experiences and behaviours $[1,2,14,15]$. If we integrate multimodal information over time, situations and events, then we can extract stable representations for experienced verbal and nonverbal items that generalise across exemplars irrespective of the situation or moment. This is one definition of "coherent" semantic concepts and has been associated with anterior temporal lobe and the end of the ventral pathways within the temporal lobe [16-18]. In a complementary, orthogonal manner, we can also integrate multimodal experiences over items, resulting in generalizable representations about order, space, number, etc. Such spatiotemporal structures are typically invariant to the elements that go into them, examples being location or number that are invariant to the items being located or counted. These types of representation have classically been associated with parietal and frontal regions along the "dorsal" pathways. Note that the input to both pathways is the same - timeextended, multimodal information - but the outcomes are mathematically orthogonal to each other 
(this is analogous to single value decomposition which generates two orthogonal similarity matrices; when applied to language texts, for example, as they are in Latent Semantic Analysis then the output includes an item/word similarity matrix and a paragraph/time-chunk similarity matrix $[19,20])$.

The second linked principle is that the local neurocomputation in this region provides the basis for online, dynamic, multi-sensory buffering that, through experience, becomes sensitive to the sequential spatio-temporal structure of an event or behaviour that unfolds over time (i.e., as a byproduct of buffering, it can extract the item-invariant representations of order, location, number, etc. noted above). The local computation is domain-general, acting on any modality (as well as multimodal combinations) of spatio-temporal input. This kind of domain-general computation is important because effective continuous verbal (e.g., speech) and nonverbal behaviours (e.g., sequential object use) require careful synchronisation of planned steps with information about the current state of the external and internal worlds [21, 22]. This information, however, arrives through different internal and external input channels and is ephemeral, necessitating a multi-modal convergent buffer or hub $[23,24]$. A number of prominent parallel distributed processing (PDP) computational models have shown that the addition of recurrent feedback loops allows a model to 'buffer' verbal or nonverbal spatiotemporal input (Elman nets: [25]) in support of time-extended verbal and nonverbal behaviours $[2,21,26]$. Furthermore, in these types of model, repeated buffering can lead to long-term statistical learning and thus by buffering time-, context- and space-varying inputs, it is possible for systems to become sensitive to content-invariant structures/schemata (indeed, the extraction of information by PDP models can be formally related to single value decomposition [27]).

The third principle is that the 'expressed' task contribution of AG, and wider LPC areas, will be influenced by their long-range connections. Thus, even on an assumption that the local buffering computation might be the same throughout the LPC, the types and forms of information being buffered will reflect the inputs and outputs to each subregion (see Box 1). Again, this principle has been formally demonstrated in various implemented computational models which have shown that the contribution of a processing unit to each task is shaped not only by its local computation but also by its connectivity to different sources of input/output information (more recently referred to as 'connectivity-constrained cognition - C3': [28-30]. Thus, even in a situation where the local unit computation is exactly the same, the contribution to different cognitive tasks can vary in a graded way across a layer of such units (taken to be analogous to a cortical region); units with equivalent connection to multiple inputs/outputs have a domain-general character whereas units with stronger connection to a subset of inputs/outputs will become more domain-specific in nature (i.e., becoming tuned towards the domain(s) for which those particular input/outputs are critical). 


\section{Episodic Memory}

Compared to the storied history of research examining the role of the parietal cortex in domains such as visuospatial attention and visuomotor abilities [31, 32], investigation of a putative role in episodic memory is a relatively recent development. Damage to medial parietal regions has been known for some time to result in amnesia [33,34], but virtually no studies of episodic memory following LPC lesions were published in the twentieth century. Patients with such lesions typically did not forget appointments with their neurologist, tended to be oriented in time and place, and could usually remember the names of objects shown to them a few minutes before. As such, they did not appear to be amnesic, and neurologists understandably focused on the patients' more debilitating cognitive deficits. However, advances in functional neuroimaging have led to a growing realisation that the AG makes an important contribution to episodic memory, resulting in an explosion of research over the last decade or so that has sought to understand what role the region might play (Figure 1).

\section{INSERT FIGURE 1 ABOUT HERE}

Research in this area was stimulated by a review of functional neuroimaging studies [35], which highlighted how left LPC responses, in particular centred on AG (mainly the PGp subregion), are closely linked with processes contributing to successful retrieval [36]. A further common finding was greater activation in AG when memory tasks involve retrieval of the context in which stimuli were previously encountered, suggesting a particular importance for conscious recollection [37]. AG appears not to contribute to successful encoding, often exhibiting deactivation [38,39], but a number of meta-analyses have confirmed the prevalence of parietal activity in the episodic retrieval fMRI literature, identifying that $\mathrm{AG}$ regions may be more consistently activated during recollection than regions traditionally considered critical for episodic memory such as the medial temporal lobes [40, $41]$.

On the basis of such findings, a number of suggestions have been made as to the functional contribution that AG might make to episodic memory. One influential review considered three possible hypotheses [35]: that AG may be involved in the allocation of attention towards aspects of mnemonic representations (a view subsequently expanded on by others [42]; see Box 2), that activity in AG regions may reflect a memory strength signal that can be used to guide behavioural responses [43], or that AG acts as a temporary storage buffer in which information retrieved from long-term memory can be maintained online $[44,45]$. It was noted that the data available at the time appeared to be partly, but not completely, explained by each of these hypotheses [35]. Subsequent findings from 
neuroimaging research, and from studies involving neuropsychological and neurostimulation-induced brain lesions, have led to the development of further theories, all of which account for some aspects of the data but fail to accommodate the full range of findings from episodic memory research, or indeed from the numerous other cognitive domains to which AG appears to contribute. These theories do, however, converge on a number of common fundamental principles, namely that the computations undertaken by AG result in dynamic, multimodal, consciously-accessible representations which integrate features of events that unfold over time.

Although LPC lesions do not result in amnesia, memory is not entirely unaffected. Two patients with bilateral parietal damage were reported to be impaired at freely recalling autobiographical memories, although their memories for the events tested appeared to be preserved when their recall was cued by specific questions [46]. This deficit in the free, but not cued, recall of autobiographical memories has been replicated by subsequent brain stimulation studies that have disrupted LPC function $[47,48]$, and found to be specific to free recall of autobiographical memories, with free recall of previously studied word-pairs, or word-definition pairs, unaffected [47, 49, 50]. The retrieval of autobiographical and word-pair memories differs in that the former often involves the subjective experience of remembering multifaceted events that take place over a sequence of phases, combining features that may be of several modalities and recalled from a first-person point-of-view [51-53]. Accordingly, AG has been found to be differentially sensitive to the retrieval of multi-modal spatio-temporal memories compared to those involving only a single sensory modality [54-57], and to integrate these features within an egocentric perspective $[47,58]$, in a way that is characteristic of true episodic memory [59].

One question is what the adaptive value might be of the kind of dynamic, consciouslyaccessible mnemonic representation that AG appears to be involved in constructing. One possibility is that subjective experience (also called "autonoetic awareness" [59]) allows us to reflect on the content of our memories, to integrate them with prior semantic knowledge, and to make judgments about the things we remember. Consistent with this view, although patients with parietal lesions perform well on many tests of episodic memory, their accurate recollections are associated with reduced confidence $[60,61]$ and fewer 'remember' responses on remember/know tasks $[49,62]$. Functional imaging experiments have found AG activity to be sensitive to qualitative characteristics of retrieved memories, such as their rated vividness, confidence, and precision [55, 63, 64]. Thus, it may be that this region contributes to episodic memory by enabling the online, dynamic buffering of multi-sensory spatio-temporally extended representations that are accessible to conscious assessment and evaluation by other, primarily prefrontally-mediated, brain networks responsible for monitoring and decision making [65-67]. The flexible coordination of such whole-brain networks that involve prefrontal, medial temporal, and parietal cortices, appears to be important for promoting successful 
recollection and the adaptive benefits that can be gained by reflecting on our memories and using them to guide subsequent behaviour [68-70].

\section{$\underline{\text { Semantic Memory }}$}

The association of the lateral parietal area with semantic processing has a long history. Henry Head [71] was one of the first to document that temporo-parietal damage leads to a form of semantic impairment known as semantic aphasia. Intriguingly for our consideration of the LPC's contribution to episodic and semantic memory, Head also noted that these same patients had difficulty in relating their personal autobiographical history (perhaps prefiguring the findings of others [46] - see above). Later, it was proposed that the AG is a "semantic hub" that stores multimodal semantic information based on neuropsychological evidence of semantic impairment without other aphasic symptoms after AG damage [72, 73]. Several large-scale, functional neuroimaging meta-analyses have shown that the ventral AG (in the PGp subregion) reliably exhibits differential activation levels with respect to semantic contrasts such as words vs. non-words, or concrete vs. abstract words [74, 75]. It is also possible that the AG semantic-hub theory could be extended to explain AG engagement in the construction and expression of autobiographical and episodic memories in that many of the constituents are semantic in nature [76]. Similar recent proposals include that common AG activation for semantic and episodic tasks reflect the "reinstatement" of conceptual processing necessary during episodic retrieval [11], or the convergence of multi-modal representations [10].

Despite the long-standing prominence of the semantic hub hypothesis and the robustness of one line of neuroimaging data, there are several important caveats and apparent contradictions. First, in terms of the neuropsychological evidence, patients with semantic aphasia do not appear to have lost semantic representations but rather have dysfunction in the flexible use and manipulation of semantic information [77]. Secondly, in revisiting a seminal case-study [73] from a contemporary viewpoint, it is striking that the patient's anoxia-induced damage was not isolated to the AG but encompassed multiple areas, including prefrontal cortex and was particularly severe in the lateral and ventral anterior temporal lobes, bilaterally - all areas known to be crucial for representing and manipulating conceptual knowledge [16].

There are also important caveats to the neuroimaging evidence, which varies dramatically depending on the contrast. Specifically, the vast majority of tasks based on word/nonword or concrete/abstract comparisons tend to involve a contrast of an easier vs. more difficult task. When studies use different contrasts then ventral AG activation is often missing. For instance, no ventral AG activation was found in a meta-analysis of semantic vs. non-semantic tasks [78] or in a study specifically directed to examine semantic involvement in AG processing, which instead found stronger activation for tongue movements than meaningful speech [79]. It is, in fact, possible that the 
very reliable differences found for concrete/abstract or word/nonword contrasts represent difficultyrelated de-activations in the ventral AG [1,7]. For example, a recent investigation that directly manipulated task difficulty for both a semantic and a non-semantic visuospatial task found a main effect of task difficulty (easy vs. hard) in the ventral AG but no semantic vs. non-semantic difference [7]. Finally and compellingly, the classic pattern of differential activation associated with the contrast of words vs. non-words or concrete vs. abstract processing, can be flipped by reversing the difficulty of the task or the stimuli $[80,81]$.

A second important issue when considering the neuroimaging literature on the AG is that it is important to take into account the polarity of activation relative to a resting baseline [1, 12]. It is, of course, always difficult to interpret 'rest', which could involve spontaneous language and semantic processing [82], however, if we consider the pattern of AG activation and deactivations across tasks, then clear differences emerge. First, as noted above for both semantic and non-semantic tasks (e.g., judgements of word meaning, episodic encoding, visuospatial decisions), the ventral AG deactivates indeed, the ventral AG forms a core part of the default mode network [83] which is not true of other brain regions known to be involved in semantic representation (e.g., the ATL shows activation for semantic tasks over rest but deactivation with non-semantic tasks [7, 12]. The ventral AG, however, is most commonly engaged positively in studies that examine episodic memory retrieval, as described above [35, 84].

Taken together, these neuropsychological and functional neuroimaging data appear to reject the classical notion that the AG supports semantic representation per se (see Figure 2 for summary of the alternative interpretations of the "semantic" ATL vs. AG hubs). The current literature involves investigation of at least two alternative hypotheses. One proposal has reformulated the classical semantic AG hypothesis to suggest, instead, that the AG might support "event-semantic" information and the ATL acts as a hub for other aspects of semantics [76]. Various forms of evidence have been used to support this emerging hypothesis, including combinatorial semantic tasks where readilycombined adjective-noun or adjective-verb word pairs elicit stronger AG activation compared to less easily combined alternatives $[85,86]$. Like the prior AG general-semantic view, this hypothesis offers an explanation for the involvement of AG in episodic retrieval by arguing that episodic memories necessitate event knowledge [76]. Challenges to this view include: (a) most of the combinatorial noun-phrase experiments are again based on contrasting an easy vs. hard task condition, which we now know can generate a difference in the ventral AG even for non-verbal, non-semantic activities [7], and (b) the multi-hub hypothesis does not appear to fit with the data from patients with semantic dementia, which arises from atrophy centred on the ATL bilaterally and not the AG, who have impoverished generalised semantic impairment including reduced knowledge about events and social schema [87]. 


\section{INSERT FIGURE 2 ABOUT HERE}

The second alternative hypothesis is that the AG does not support long-term stored information per se but rather is a multi-modal online temporary buffer for external and internal information. Indeed, this hypothesis might be consistent with other proposals that AG acts a "schematic-convergence zone" which binds information, if we assume that this binding is temporary [88]. An online buffer would seem to be a necessary neurocomputation for the construction of internal models of the world, reconstruction of autobiographical memories, or the envisioning of possible future events, and, perhaps, for the ongoing buffering of combinatorial meaning generated over a time-extended period [89-91]. All these processes would require the positive engagement of the AG whereas the activation of semantic representations via the hub-and-spoke architecture does not require online buffering and thus the AG is not engaged or even deactivated. When this form of AG buffering system is damaged, then neuropsychological patients should find it difficult to construct detailed autobiographical memories, and to complete other time-extended activities that all require an online internal buffering of recent stimuli and events.

\section{Concluding Remarks}

As siblings within the 'declarative' or 'explicit' family of long-term memory systems, episodic and semantic memory are defined by many shared features in addition to their essential differences. Despite the closeness of their taxonomic relationship, the cognitive and brain mechanisms of these two forms of memory have largely been investigated independently, leading to a proliferation of theories which overlook that episodic and semantic memory (as well as a broader range of other non-memory cognitive activities) engage a number of the same brain areas, and thus presumably share at least some underlying neurocomputations. One common brain area is AG, classically implicated in both episodic and semantic memory, as well as several other cognitive domains. Building on previous domain-specific theories of parietal function, we have here considered a unifying model that encompasses a set of common principles arising from cognitive neuroscience. This cross-domain synthesis proposes that AG, and wider LPC, computations support the online dynamic buffering that combines distinct forms of information, such as multiple sensory modalities or different spatio-temporal frameworks. Further work is required to understand the purpose and implications of such an integrative buffering function (see Outstanding Questions). Its uses might include the internal representation of the current external and internal state of the world which would seem to be a necessary element for keeping track of time-extended events or activities. With respect to 
higher forms of human cognitive functions, the AG buffer may enable the conscious evaluation and exploration of the complex feature networks that comprise many of our autobiographical experiences and conceptual knowledge structures. Such in-depth and demanding cognitive processing may not be necessary for accomplishing many laboratory memory tasks, such as those that require the retrieval of a single episodic feature or semantic fact. But the capacity to flexibly and dynamically reflect on the content of our memories affords us the invaluable real-world ability to understand and learn from our experiences, and use them to guide subsequent action in novel and creative ways that may be crucial for successful adaptive human behaviour. 


\section{Box 1. Functional heterogeneity of lateral parietal cortex}

The LPC is a structurally and functionally heterogeneous region, with multiple cytoarchtectonic subdivisions [92]. There appear to be two primary axes of variation; a dorsal vs. ventral gradient, and an anterior vs. posterior gradient (although boundaries are graded rather than a sharply fractionated [93]). The dorsal IPL (dIPL) forms part of a fronto-parietal system, whereas vIPL connects with a distributed set of regions associated with the default mode network or saliency network [68, 94-100]. In terms of function, dIPL forms part of a domain general fronto-parietal multiple demand network engaged by tasks that require a degree of executive control (working memory, numerical calculation, top-down attention) [101]. Whereas within vIPL, SMG is associated with tasks involving phonological processes, theory of mind, and bottom up attention [1].

It is possible that a wide variety of cognitive activities can arise from a single underlying machinery, with distinctions in emergent functions across the region arising based on graded variation in connectivity. Even if the local buffering computation is the same throughout the LPC, the types and forms of information being buffered will reflect the inputs and outputs to each subregion. Applying this generalised hypothesis to LPC provides a potential explanation for the contrastive (and sometimes anticorrelated) expressed characteristics of various subregions. Specifically, dIPL is structurally connected to frontal executive processing areas [94, 102]. Most notions of executive control and attention require the action of selection/manipulation processes on internally-buffered information (akin to a working memory system), which might be reflected in prefrontal regions sending top-down signals to dIPL, as demonstrated in primate electrophysiological studies [103]. In contrast, without the direct influence of prefrontal goal-directed cognition, the vIPL will act more like a 'slave' buffer whereby information is accumulated and maintained throughout a sequential activity. In addition to the emergent dorsal-ventral connectivity/functional differences, there are known anterior-posterior variations within the vIPL and it is possible that these subdivisions also emerge from differential connectivity to separate networks for language, memory, visuospatial processing, etc. [93, 94, 102]. A dorsal-ventral subdivision can be observed within AG itself. Specifically, dorsal AG (PGa)/lateral bank of intraparietal sulcus (IPS) serves a distinctive function to the ventral AG (central PGp), which is commonly associated with the default mode network. Indeed, dorsal and ventral AG showing opposing effect of task difficulty in semantic and visuo-spatial tasks; dorsal areas show a greater response when difficulty is increased, whereas ventral AG shows the inverse pattern - stronger deactivation for harder tasks [7], as discussed in the main text. Dorsal and ventral AG also play distinct roles in episodic memory tasks, dorsal AG/IPS acts as a "mnemonic accumulator" that guides episodic decisions, whereas ventral AG is (positively) engaged by episodic recollection [104, 105]. 


\section{Box 2. Attention Models}

Dorsal vs. ventral parietal cortex: The current review focuses on the contribution of LPC to episodic and semantic memory, but a discussion of parietal function cannot be complete without a mention of the attention literature. One seminal attention model [13] proposed that LPC is divided into dorsal and ventral areas, which participate in top-down vs. bottom-up/stimulus driven attention, respectively. According to this model, the stimulus-driven network acts as an alerting system, or "circuit breaker", for top-down processing. Some kind of dorsal-ventral LPC distinction is broadly recognised across investigations and theories of many different cognitive domains. For instance, dorsal IPL/IPS forms a key region of the executive processing network responding more strongly to difficult decisions or task demands across diverse domains and task types $[6,7,101]$. The ventral LPC is involved in bottomup/stimulus-driven and automatic task components [1,8]. Greater AG activation is associated with faster reaction times [106] and is more sensitive to automated than executively demanding tasks: e.g., numerical fact retrieval versus numerical calculation, or making semantic decisions on concrete versus abstract words [1]. Indeed, there is also a dorsal-ventral distinction within the episodic memory literature, whereby "recollection" is associated with AG responses and "familiarity" with the IPS [35].

Attention in the ventral parietal cortex: In some of the first attempts to unify explanations of LPC function across cognitive domains, it was proposed that the bottom-up attention system is not only automatically captured by salient external stimuli (e.g., a loud noise) but also salient internal information, such as when an episodic memory is triggered and readily "pops" into awareness [42]. Much evidence supports the view that ventral LPC plays an attentional role in episodic memory [8], although other findings question predictions of this model. For example, neuropsychology and neurostimulation studies targeting ventral LPC report no disproportionate impairments in episodic memory tasks that involve attentional manipulations $[47,50,61,107])$. Although attention and memory can both be associated with bilateral LPC activity, episodic retrieval effects are typically observed in the left AG, whereas attentional reorientation is generally associated with the more anterior temporo-parietal junction (TPJ) area in the right hemisphere [1, 108, 109]. This implies that there may be a separation of cognitive functions both within and across the left and right ventral LPC. 


\section{References}

1 Humphreys, G.F. and Lambon Ralph, M.A. (2015) Fusion and fission of cognitive functions in the human parietal cortex. Cerebral Cortex 25, 3547-3560

2 Ueno, T., et al. (2011) Lichtheim 2: Synthesizing Aphasia and the Neural Basis of Language in a Neurocomputational Model of the Dual Dorsal-Ventral Language Pathways. Neuron 72, 385-396 3 Seidenberg, M.S. and McClelland, J.L. (1989) A distributed, developmental model of word recognition and naming. Psychol Rev 96, 523-568

4 Patterson, K. and Lambon Ralph, M.A. (1999) Selective disorders of reading? Current Opinion in Neurobiology 9, 235-239

5 Cabeza, R., et al. (2018) Process-Specific Alliances (PSAs) in Cognitive Neuroscience. Trends Cogn Sci 22, 996-1010

6 Noonan, K.A., et al. (2013) Going beyond inferior prefrontal involvement in semantic control: evidence for the additional contribution of dorsal angular gyrus and posterior middle temporal cortex. J Cognitive Neurosci 25, 1824-1850

7 Humphreys, G.F. and Lambon Ralph, M.A. (2017) Mapping Domain-Selective and Counterpointed Domain-General Higher Cognitive Functions in the Lateral Parietal Cortex: Evidence from fMRI Comparisons of Difficulty-Varying Semantic Versus Visuo-Spatial Tasks, and Functional Connectivity Analyses. Cereb Cortex 27, 4199-4212

8 Cabeza, R., et al. (2012) Cognitive contributions of the ventral parietal cortex: an integrative theoretical account. Trends Cogn Sci 16, 338-352

9 Seghier, M.L. (2013) The angular gyrus: Multiple functions and multiple subdivisions. The Neuroscientist 19, 43-61

10 Rugg, M.D. and King, D.R. (2018) Ventral lateral parietal cortex and episodic memory retrieval. Cortex 107, 238-250

11 Renoult, L., et al. (2019) From Knowing to Remembering: The Semantic-Episodic Distinction. Trends Cogn Sci 23, 1041-1057

12 Humphreys, G.F., et al. (2015) Establishing task- and modality-dependent dissociations between the semantic and default mode networks. P Natl Acad Sci USA 112, 7857-7862

13 Corbetta, M. and Shulman, G.L. (2002) Control of goal-directed and stimulus-driven attention in the brain. Nat Rev Neurosci 3, 201-215

14 Bornkessel-Schlesewsky, I. and Schlesewsky, M. (2013) Reconciling time, space and function: A new dorsal-ventral stream model of sentence comprehension. Brain Lang 125, 60-76

15 Bornkessel-Schlesewsky, I., et al. (2015) Neurobiological roots of language in primate audition: common computational properties. Trends Cogn Sci 19, 142-150

16 Lambon Ralph, M.A., et al. (2017) The neural and computational bases of semantic cognition. Nat Rev Neurosci 18, 42-55 
17 Lambon Ralph, M.A., et al. (2010) Coherent concepts are computed in the anterior temporal lobes. P Natl Acad Sci USA 107, 2717-2722

18 Mesulam, M.M. (1998) From sensation to cognition. Brain 121, 1013-1052

19 Landauer, T.K. and Dumais, S.T. (1997) A solution to Plato's problem: The latent semantic analysis theory of acquisition, induction, and representation of knowledge. Psychol Rev 104, 211-240 20 Hoffman, P., et al. (2013) Semantic diversity: A measure of semantic ambiguity based on variability in the contextual usage of words. Behavior research methods 45, 718-730 21 Botvinick, M.M. and Plaut, D.C. (2004) Doing without schema hierarchies: A recurrent connectionist approach to normal and impaired routine sequential action. Psychol Rev 111, 395-429 22 Botvinick, M.M. and Plaut, D.C. (2006) Short-term memory for serial order: A recurrent neural network model. Psychol Rev 113, 201-233

23 Geschwind, N. (1965) Disconnexion syndromes in animals and man. Brain 88, 237-294

24 Damasio, A.R. (1989) Time-locked multiregional retroactivation: A systems-level proposal for the neural substrates of recall and recognition. Cognition 33, 25-62

25 Elman, J.L. (1990) Finding Structure in Time. Cognitive Science 14, 179-211

26 McClelland, J.L., et al. (1989) Sentence comprehension: A parallel distributed processing approach. Language and Cognitive Processes 4, 287-335

27 Saxe, A.M., et al. (2019) A mathematical theory of semantic development in deep neural networks. P Natl Acad Sci USA 116, 11537-11546

28 Chen, L., et al. (2017) A unified model of human semantic knowledge and its disorders. Nat Hum Behav 1, 1-10

29 Lambon Ralph, M.A., et al. (2001) No right to speak? The relationship between object naming and semantic impairment: neuro- psychological evidence and a computational model. J Cognitive Neurosci 13, 341-356

30 Plaut, D.C. (2002) Graded modality-specific specialization in semantics: A computational account of optic aphasia. Cognitive Neuropsychology 19, 603-639

31 Mesulam, M.M. (1999) Spatial attention and neglect: Parietal, frontal and cingulate contributions to the mental representation and attentional targeting of salient extrapersonal events. Philosophical Transactions of the Royal Society of London, Series B: Biological Sciences 354, 1325-1346

32 Milner, A.D. and Goodale, M.A. (1995) The visual brain in action. OUP

33 Cavanna, A.E. and Trimble, M.R. (2006) The precuneus: A review of its functional anatomy and behavioural correlates. Brain 129, 564-583

34 Valenstein, E., et al. (1987) Retrosplenial amnesia. Brain 110, 1631-1646

35 Wagner, A.D., et al. (2005) Parietal lobe contributions to episodic memory retrieval. Trends Cogn Sci 9, 445-453

36 Konishi, S., et al. (2000) Neural correlates of episodic retrieval success. Neuroimage 12, 276-286 
37 Henson, R.N.A., et al. (1999) Recollection and familiarity in recognition memory: An eventrelated functional magnetic resonance imaging study. J Neurosci 19, 3962-3972

38 Otten, L.J. and Rugg, M.D. (2001) When more means less: Neural activity related to unsuccessful memory encoding. Current Biology 11, 1528-1530

39 Wagner, A.D. and Davachi, L. (2001) Cognitive neuroscience: Forgetting of things past. Current Biology 11, 964-967

40 Simons, J.S., et al. (2008) Is the parietal lobe necessary for recollection in humans?

Neuropsychologia 46, 1185-1191

41 Skinner, E.I. and Fernandes, M.A. (2007) Neural correlates of recollection and familiarity: A review of neuroimaging and patient data. Neuropsychologia 45, 2163-2179

42 Cabeza, R., et al. (2008) The parietal cortex and episodic memory: an attentional account. Nature Reviews Neuroscience 9, 613-625

43 Wheeler, M.E. and Buckner, R.L. (2003) Functional dissociations among components of remembering: Control, perceived oldness, and content. J Neurosci 23, 3869-3880

44 Baddeley, A. (2000) The episodic buffer: A new component of working memory? Trends Cogn Sci 4, 417-423

45 Vilberg, K.L. and Rugg, M.D. (2008) Memory retrieval and the parietal cortex: A review of evidence from a dual-process perspective. Neuropsychologia 46, 1787-1799

46 Berryhill, M.E., et al. (2007) Parietal lobe and episodic memory: Bilateral damage causes impaired free recall of autobiographical memory. J Neurosci 27, 14415-14423

47 Bonnici, H.M., et al. (2018) Specifying a causal role for angular gyrus in autobiographical memory. J Neurosci 38, 10438-10443

48 Thakral, P.P., et al. (2017) A role for the left angular gyrus in episodic simulation and memory. $J$ Neurosci 37, 8142-8149

49 Davidson, P.S.R., et al. (2008) Does lateral parietal cortex support episodic memory? Evidence from focal lesion patients. Neuropsychologia 46, 1743-1755

50 Yazar, Y., et al. (2014) Continuous theta burst stimulation of angular gyrus reduces subjective recollection. Plos One 9, e110414

51 Moscovitch, M., et al. (2016) Episodic memory and beyond: The hippocampus and neocortex in transformation. Annual Review of Psychology 67, 105-134

52 Shimamura, A.P. (2011) Episodic retrieval and the cortical binding of relational activity. Cogn Affect Behav Neurosci 11, 277-291

53 St. Jacques, P.L. (2019) A New Perspective on Visual Perspective in Memory. Current Directions in Psychological Science, 0963721419850158

54 Bonnici, H.M., et al. (2016) Multimodal feature integration in the angular gyrus during episodic and semantic retrieval. $J$ Neurosci 36, 5462-5471 
55 Richter, F.R., et al. (2016) Distinct neural mechanisms underlie the success, precision, and vividness of episodic memory. ELife

56 Yazar, Y., et al. (2017) Reduced multimodal integration of memory features following continuous theta burst stimulation of angular gyrus. Brain Stimulation 10, 624-629

57 Ben-Zvi, S., et al. (2015) Parietal lesion effects on cued recall following pair associate learning. Neuropsychologia 73, 176-194

58 Russel, C., et al. (2019) Self-perspective in episodic memory after parietal damage and in healthy ageing. Neuropsychologia 124, 171-181

59 Tulving, E. (1983) Elements of Episodic Memory. Clarendon Press

60 Hower, K.H., et al. (2014) Impaired perception of mnemonic oldness, but not mnemonic newness, after parietal lobe damage. Neuropsychologia 56, 409-417

61 Simons, J.S., et al. (2010) Dissociation between memory accuracy and memory confidence following bilateral parietal lesions. Cerebral Cortex 20, 479-485

62 Drowos, D.B., et al. (2010) True memory, false memory, and subjective recollection deficits after focal parietal lobe lesions. Neuropsychologia 24, 465-475

63 Kuhl, B.A. and Chun, M.M. (2014) Successful remembering elicits event-specific activity patterns in lateral parietal cortex. $J$ Neurosci 34, 8051-8060

64 Tibon, R., et al. (2019) Multimodal integration and vividness in the angular gyrus during episodic encoding and retrieval. J Neurosci 39, 4365-4374

65 Badre, D. and Wagner, A.D. (2007) Left ventrolateral prefrontal cortex and the cognitive control of memory. Neuropsychologia 45, 2883-2901

66 Simons, J.S., et al. (2017) Brain mechanisms of reality monitoring. Trends Cogn Sci 21, 462-473

67 Simons, J.S. and Spiers, H.J. (2003) Prefrontal and medial temporal lobe interactions in long-term memory. Nature Reviews Neuroscience 4, 637-648

68 Spreng, R.N., et al. (2010) Default network activity, coupled with the frontoparietal control network, supports goal-directed cognition. Neuroimage 53, 303-317

69 Fornito, A., et al. (2012) Competitive and cooperative dynamics of large-scale brain functional networks supporting recollection. P Natl Acad Sci USA 109, 12788-12793

70 Westphal, A.J., et al. (2017) Episodic memory retrieval benefits from a less modular brain network organization. J Neurosci 37, 3523-3531

$71 \mathrm{Head}$, H. (1926) Aphasia and kindred disorders of speech. Cambridge University Press

72 Geschwind, N. (1972) Language and Brain. Sci Am 226, 76-\&

73 Geschwind, N., et al. (1968) Isolation of Speech Area. Neuropsychologia 6, 327-+

74 Binder, J.R., et al. (2009) Where Is the Semantic System? A Critical Review and Meta-Analysis of 120 Functional Neuroimaging Studies. Cerebral Cortex 19, 2767-2796

75 Wang, J., et al. (2010) Neural Representation of Abstract and Concrete Concepts: A MetaAnalysis of Neuroimaging Studies. Hum Brain Mapp 31, 1459-1468 
76 Binder, J.R. and Desai, R.H. (2011) The neurobiology of semantic memory. Trends Cogn Sci 15, $527-536$

77 Jefferies, E. and Lambon Ralph, M.A. (2006) Semantic impairment in stroke aphasia versus semantic dementia: a case-series comparison. Brain 129, 2132-2147

78 Visser, M., et al. (2010) Semantic Processing in the Anterior Temporal Lobes: A Meta-analysis of the Functional Neuroimaging Literature. J Cognitive Neurosci 22, 1083-1094

79 Geranmayeh, F., et al. (2012) The contribution of the inferior parietal cortex to spoken language production. Brain Lang 121, 47-57

80 Pexman, P.M., et al. (2007) Neural correlates of concreteness in semantic categorization. $J$ Cognitive Neurosci 19, 1407-1419

81 Graves, W.W., et al. (2017) Reversing the Standard Neural Signature of the Word-Nonword Distinction. J Cognitive Neurosci 29, 79-94

82 Binder, J.R., et al. (1999) Conceptual processing during the conscious resting state: A functional MRI study. J Cognitive Neurosci 11, 80-93

83 Buckner, R.L., et al. (2008) The brain's default network - Anatomy, function, and relevance to disease. Ann Ny Acad Sci 1124, 1-38

84 Vilberg, K.L. and Rugg, M.D. (2012) The Neural Correlates of Recollection: Transient Versus Sustained fMRI Effects. J Neurosci 32, 15679-15687

85 Matchin, W., et al. (2019) Same words, different structures: An fMRI investigation of argument relations and the angular gyrus. Neuropsychologia 125, 116-128

86 Bemis, D.K. and Pylkkanen, L. (2013) Basic Linguistic Composition Recruits the Left Anterior Temporal Lobe and Left Angular Gyrus During Both Listening and Reading. Cerebral Cortex 23, $1859-1873$

87 Lambon Ralph, M.A. and Patterson, K. (2008) Generalization and differentiation in semantic memory. Annals of the New York Academy of Sciences 1124, 61-76

88 Wagner, I.C., et al. (2015) Schematic memory components converge within angular gyrus during retrieval. ELife 4, e09668

89 Hasson, U., et al. (2008) A hierarchy of temporal receptive windows in human cortex. J Neurosci $28,2539-2550$

90 Lerner, Y., et al. (2011) Topographic Mapping of a Hierarchy of Temporal Receptive Windows Using a Narrated Story. J Neurosci 31, 2906-2915

91 Ramanan, S. and Bellana, B. (2019) A domain-general role for the angular gyrus in retrieving internal representations of the external world. J Neurosci 39, 2978-2980

92 Caspers, S., et al. (2008) The human inferior parietal lobule in stereotaxic space. Brain Struct Funct 212, 481-495

93 Daselaar, S.M., et al. (2013) Resting- state functionl connectivity of ventral parietal regions associated with attention reorienting and episodic recollection. Front Hum Neurosci 7 
94 Cloutman, L.L., et al. (2013) Using in vivo probabilistic tractography to reveal two segregated dorsal 'language-cognitive' pathways in the human brain. Brain Lang 127, 230-240

95 Power, J.D. and Petersen, S.E. (2013) Control-related systems in the human brain. Current Opinion in Neurobiology 23, 223-228

96 Vincent, J.L., et al. (2008) Evidence for a frontoparietal control system revealed by intrinsic functional connectivity. Journal of Neurophysiology 100, 3328-3342

97 Uddin, L.Q., et al. (2010) Dissociable Connectivity within Human Angular Gyrus and Intraparietal Sulcus: Evidence from Functional and Structural Connectivity. Cerebral Cortex 20, 2636-2646 98 Lee, M.H., et al. (2012) Clustering of resting state networks. Plos One 7, e40370

99 Yeo, B.T., et al. (2013) Estimates of segregation and overlap of functional connectivity networks in the human cerebral cortex. Neuroimage 88C, 212-227

100 Power, J.D., et al. (2011) Functional network organization of the human brain. Neuron 72, 665678

101 Fedorenko, E., et al. (2013) Broad domain generality in focal regions of frontal and parietal cortex. Proc Natl Acad Sci U S A 110, 16616-16621

102 Caspers, S., et al. (2011) Probabilistic fibre tract analysis of cytoarchitectonically defined human inferior parietal lobule areas reveals similarities to macaques. Neuroimage 58, 362-380

103 Crowe, D.A., et al. (2013) Prefrontal neurons transmit signals to parietal neurons that reflect executive control of cognition. Nat Neurosci 16, 1484-1491

104 Sestieri, C., et al. (2017) The contribution of the human posterior parietal cortex to episodic memory. Nature Reviews Neuroscience 18, 183-192

105 Gonzalez, A.J., et al. (2015) Parietal dynamics during recognition memory. P Natl Acad Sci USA 112, 11066-11071

106 Hahn, B., et al. (2007) Cingulate activation increases dynamically with response speed under stimulus unpredictability. Cerebral Cortex 17, 1664-1671

107 Dobbins, I.G., et al. (2012) Use of explicit memory cues following parietal lobe lesions.

Neuropsychologia 50, 2992-3003

108 Nelson, S.M., et al. (2012) In favor of a 'fractionation' view of ventral parietal cortex: comment on Cabeza et al. Trends Cogn Sci 16, 399-400; author reply 400-391

109 Hutchinson, J.B., et al. (2009) Posterior parietal cortex and episodic retrieval: Convergent and divergent effects of attention and memory. Learning and Memory 16, 343-356 

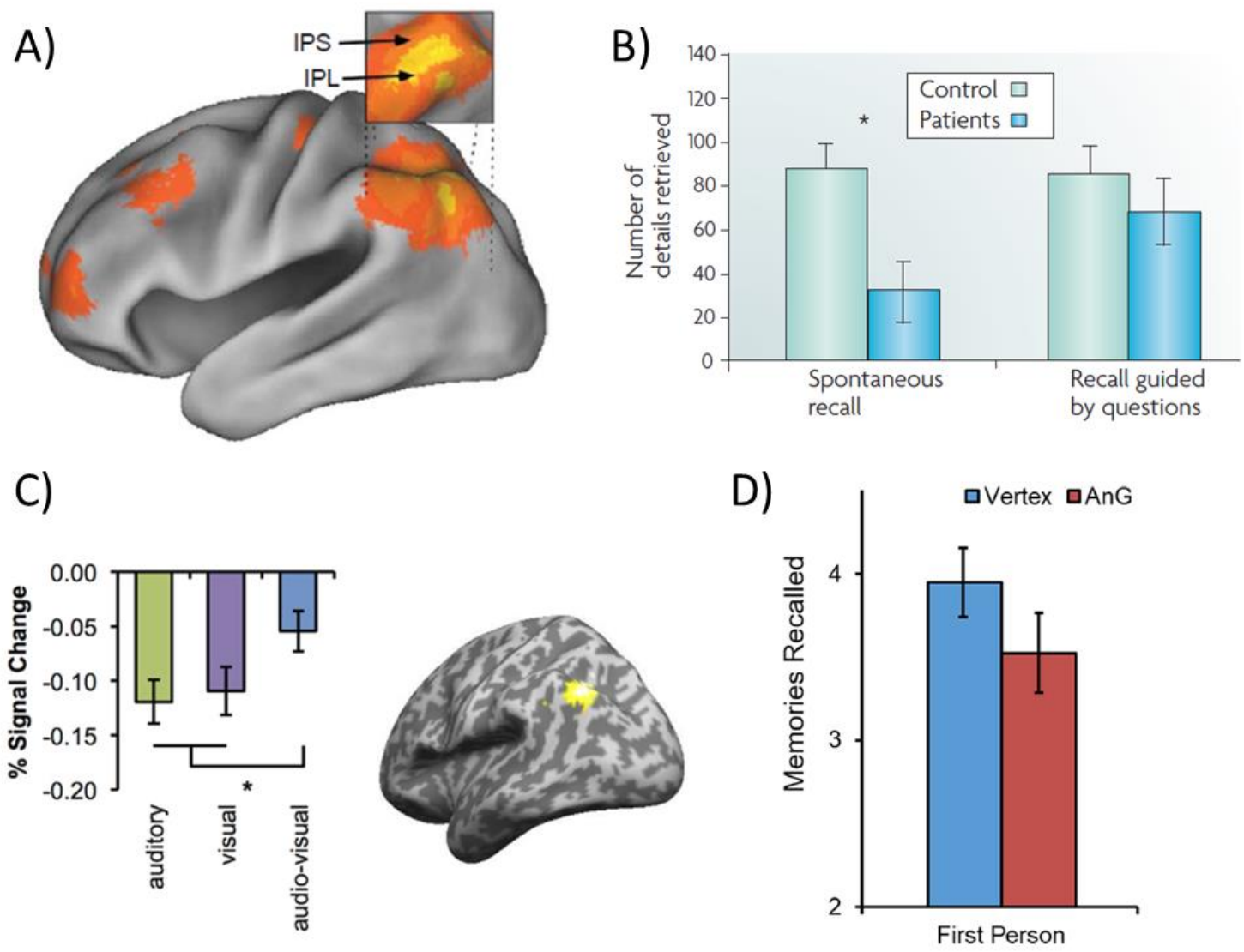

Figure 1. Role of AG in episodic memory may be to enable the subjective experience of remembering. The results in Panel $A$ highlight consistent LPC activity in neuroimaging studies of episodic retrieval, particularly around IPL regions such as the angular gyrus (AG) [35]. Panel B shows results from a study testing autobiographical memory in patients with bilateral IPL lesions [46]. The patients were impaired when freely recalling events from their lifetimes but their memory was unimpaired when answering specific questions about the events. Several studies have identified that AG is sensitive to retrieval of multimodal episodic memories. The results shown in Panel $C$ are one example, finding that AG exhibits greater activity during retrieval of integrated audio-visual compared to unimodal information [54]. Another feature of IPL function may be in integrating retrieved episodic features within an egocentric perspective. Data shown in Panel D illustrate how disrupting AG with brain stimulation results in fewer autobiographical memories being reported as experienced from a firstperson perspective [47]. Together these results converge on the notion that AG computations drive the online, dynamic buffering of multi-sensory spatio-temporally extended representations that can enable the subjective experience of remembering past events. 


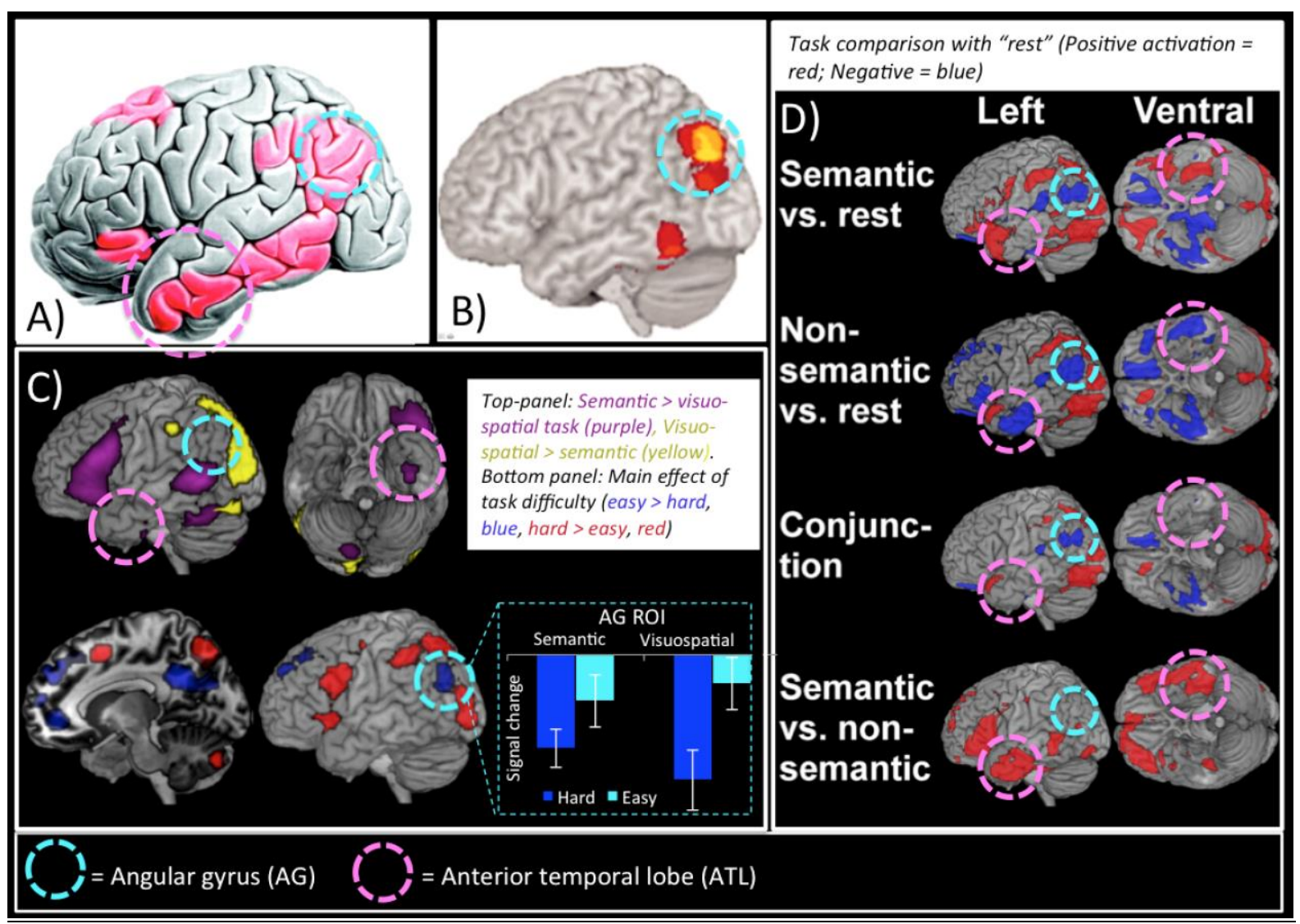

Figure 2. Alternative interpretations of the 'semantic' ATL vs. AG hubs. The results from Panels $A$ and $B$ have both been interpreted as strong evidence that the AG is a core semantic hub. Specifically, Panel A shows the summary from a meta-analysis [74] that included studies using a semantic contrast (e.g., words vs. non-words, concrete $>$ abstract items). Panel B shows the results from a meta-analysis which contrasted concrete $>$ abstract items [75]. Nevertheless, unlike areas such as the positivelyactivated posterior inferior temporal gyrus (ITG), the results in the AG largely reflected differential levels of deactivation, which raises the alternative possibility that they result from differential difficulty rather than semantic representation. This is clearly illustrated in the results from Panel D [12]. This study showed that, when contrasting tasks vs. a resting baseline, the AG is consistently deactivated for both semantic studies > rest, and non-semantic tasks > rest (blue regions). Importantly, there is no difference in AG activity levels when directly comparing the semantic and non-semantic studies. This is quite unlike the core ATL semantic hub, which is positively activated for semantic tasks (red activation), but deactivated for non-semantic control tasks.

It is important to realise that AG deactivation can, for many though not all tasks, vary in line with task or item difficulty. For instance, in common "semantic" contrasts there is often a difficulty confound: in contrasts such as words > non-words, or concrete > abstract, the easier task is contrasted with the harder task. In fact, the results from these contrasts can be reversed by manipulating task/item difficulty to be in the opposite direction [80,81]. The importance of difficulty-related deactivations is demonstrated in Panel $C$. This study directly manipulated difficulty of semantic and visuo-spatial tasks. Again, when semantic > non-semantic contrasts are matched in task difficulty (purple regions) [7], the AG does not show a response (whereas other known-semantic regions do respond, including the ATL). Strikingly, the AG shows a task difficulty effect, revealing the same easy > hard difference not only in semantic but also non-semantic, visuospatial decisions (blue areas). 